\title{
Quasi-spherical accretion in low-luminosity X-ray pulsars: Theory vs. observations
}

\author{
K. Postnov ${ }^{* a}$, N. Shakura ${ }^{a}$, A. Kochetkova ${ }^{a}$, L. Hjalmarsdotter ${ }^{a}$ \\ ${ }^{a}$ Moscow M.V. Lomonosov State University, Sternberg Astronomical Institute, 13, Universitetskij \\ pr., 119992 Moscow, Russia \\ E-mail: kpostnov@gmail.com, nikolai.shakura@gmail.com, \\ apostnova@mail.com, astrogirletelia.com
}

\begin{abstract}
Quasi-spherical subsonic accretion can be realized in slowly rotating wind-fed X-ray pulsars at moderate and small X-ray luminosities $L_{x} \lesssim 4 \times 10^{36} \mathrm{erg} / \mathrm{s}$. In this regime the accreting matter settles down subsonically onto the rotating magnetosphere, forming an extended quasi-static shell. The shell mediates the angular momentum removal from the rotating neutron star magnetosphere by shear turbulent viscosity in the boundary layer near the magnetosphere or via large-scale convective motions. In the last case the differential rotation law in the shell is close to iso-angularmomentum rotation with $\omega \sim 1 / R^{2}$. The accretion rate through the shell is determined by the ability of the plasma to enter the magnetosphere due to Rayleigh-Taylor instabilities while taking cooling into account. Measurements of spin-up/spin-down rates of quasi-spherically wind accreting X-ray pulsars in equilibrium with known orbital periods (like e.g. GX 301-2 and Vela X-1) enable determination the main dimensionless parameters of the model, as well as the estimate of the neutron star magnetic field. For equilibrium pulsars with independent measurements of the magnetic field, the velocity of the stellar wind from the companion can be estimated without the use of complicated spectroscopical measurements. For non-equilibrium pulsars, there exists a maximum possible spin-down torque exerted on the accreting neutron star. From observations of the spin-down rate and X-ray luminosity in such pulsars (GX 1+4, SXP 1062, 4U 2206+54, etc.) a lower limit on the neutron star magnetic field is derived, which in all cases turns out to be close to the standard one and in agreement with cyclotron line measurements.
\end{abstract}

An INTEGRAL view of the high-energy sky (the first 10 years) - 9th INTEGRAL Workshop and celebration of the 10th anniversary of the - launch,

15-19 October 2012

Bibliotheque Nationale de France, Paris, France

*Speaker. 


\section{Two regimes of quasi-spherical accretion in X-ray pulsars}

There can be two different regimes of quasi-spherical accretion (see e.g. [1] for a recent review of previous studies of wind accretion). The captured stellar wind is heated up in the bow shock at Bondi radius $\sim R_{B}=2 G M / v^{2}$ (where $v$ is the relative stellar wind velocity) to high temperatures $k_{B} T \sim m_{p} v^{2}$. If the characteristic cooling time of plasma $t_{\text {cool }}$ is smaller than the free-fall time $t_{f f}=R_{B} / \sqrt{2 G M / R_{B}}$, the gas falls supersonically toward the magnetosphere with the formation of a shock. This regime is usually considered in connection with bright XPSRs [2], [3]. The role of Xray photons generated near the NS surface is two-fold: first, they heat up plasma in the bow-shock zone via photoionization, and second, they cool down the hot plasma near the magnetosphere (with $k_{B} T \sim G M / R_{A}$ ) by Compton processes thus allowing matter to enter the magnetosphere via the Rayleigh-Taylor instability [4].

In the free-fall accretion regime, the X-ray luminosity (the mass accretion rate $\dot{M}$ ) is determined by the rate of gravitational capture of stellar wind at $R_{B}$ (Bondi-Hoyle-Littleton formula, $\dot{M} \sim \rho v R_{B}^{2}$ ). The accretion torque exerted on the NS due to plasma-magnetopshere interaction is always of the same sign as the specific angular momentum of the gravitationally captured stellar wind $j_{m}$, and the NS can spin-up or spin down.

If the relative wind velocity $v$ at $R_{B}$ is slow $(\lesssim 80 \mathrm{~km} / \mathrm{s})$, the photoionization heating of plasma is important, but the radiation cooling time of plasma is shorter than the free-fall time, so the free-fall accretion regime is realized. If the wind velocity is larger than $\sim 80-100 \mathrm{~km} / \mathrm{s}$, the post-shock temperature is higher than $5 \times 10^{5} \mathrm{~K}$ (the maximum photoionization temperature for a photon temperature of several $\mathrm{keV}$ ); for $L_{x} \lesssim 4 \times 10^{36} \mathrm{erg} / \mathrm{s}$, the plasma radiative cooling time is longer than the free-fall time, so a hot quasi-spherical shell is formed above the magnetosphere with temperature determined by hydrostatic equilibrium [5], [6]. Accretion of matter through such a shell is subsonic, so no shock is formed above the magnetosphere. The accretion rate $\dot{M}$ is now determined by the ability of hot plasma to enter magnetosphere. This is the settling accretion regime.

\section{Settling accretion regime: theory}

The theory of settling accretion regime was elaborated in [6]. In this regime, the accreting matter subsonically settles down onto the rotating magnetosphere forming an extended quasi-static shell. This shell mediates the angular momentum transfer to/from the rotating NS magnetosphere by viscous stresses due to large-scale convective motions and turbulence. The settling regime of accretion can be realized for moderate accretion rates $\dot{M}<\dot{M}_{*} \simeq 4 \times 10^{16} \mathrm{~g} / \mathrm{s}$. At higher accretion rates, a free-fall gap above the neutron star magnetosphere appears due to rapid Compton cooling, and accretion becomes highly non-stationary.

The mass accretion rate through the hot shell is determined by mean velocity of matter entering the magnetosphere, $u\left(R_{A}\right)=f(u) \sqrt{2 G M / R_{A}}$. The dimensionless factor $f(u)$ is determined by the Compton cooling of plasma above magnetosphere and the critical temperature for RayleighTaylor instability to develop [4], and is found to be $f(u) \sim\left(t_{f f} / t_{c o o l}\right)^{1 / 3}$. In the case of Compton cooling

$$
f(u) \approx 0.4 \dot{M}_{16}^{4 / 11} \mu_{30}^{-1 / 11}
$$


where $\dot{M}_{16}=\dot{M} /\left[10^{16} \mathrm{~g} / \mathrm{s}\right]$ and $\mu_{30}=\mu /\left[10^{30} \mathrm{Gcm}^{3}\right]$ is the NS magnetic moment. The definition of the Alfven radius in this case is different from the value used for disk accretion:

$$
R_{A} \approx 1.6 \times 10^{9}[\mathrm{~cm}]\left(\frac{\mu_{30}^{3}}{\dot{M}_{16}}\right)^{2 / 11} .
$$

Accretion torques applied to NS in this regime are determined not only by the specific angular momentum of captured matter $j_{m} \sim \Omega_{b} R_{B}^{2}\left(\Omega_{b}\right.$ is the orbital angular velocity of the NS), as is the case of the free-fall accretion, but also by the possibility to transfer angular momentum to/from the rotating magnetosphere through the shell by large-scale convective motions. The plasmamagnetosphere interaction results in emerging of the toroidal magnetic field $B_{t} / B_{p}=\left(K_{1} / \zeta\right)\left(\omega_{m}-\right.$ $\left.\omega^{*}\right) / \omega_{K}\left(R_{A}\right)$, where $\omega_{m}$ is the angular frequency of matter at the Alfven radus, $K_{1} \sim 1$ the dimensionless coupling coefficient which is different in different sources, $\omega_{K}\left(R_{A}\right)$ is the Keplerian angular frequecy, and $\zeta$ is the size of the region of plasma-magnetopshere angular moentum coupling in units of the Alfven radius $R_{A}$. The NS spin evolution equation reads:

$$
I \dot{\omega}^{*}=\frac{K_{1}}{\zeta} K_{2} \frac{\mu^{2}}{R_{A}^{3}} \frac{\omega_{m}-\omega^{*}}{\omega_{K}\left(R_{A}\right)}+z \dot{M} R_{A}^{2},
$$

where the second term takes into acount the angular momentum braught to the NS with the infalling matter $(z<1)$. This formula can be rearraged to the form

$$
I \dot{\omega}^{*}=Z \dot{M} R_{A}^{2}\left(\omega_{m}-\omega^{*}\right)+z \dot{M} R_{A}^{2} \omega^{*},
$$

where the coupling coefficient is $Z \approx 0.36\left(K_{1} / \zeta\right) \dot{M}_{16}^{-4 / 11} \mu_{30}^{1 / 11}$.

The gas-dynamical treatment of the problem of angular momentum transfer through the shell by viscous turbulence stresses [6] showed that $\omega_{m} \approx \Omega_{b}\left(R_{A} / R_{B}\right)^{n}$, where the index $n$ depends on the character of turbulence in the shell. For example, in the case of isotropic near-sonic turbulence we obtain $n \simeq 3 / 2$, i.e. quasi-Keplerian rotation distribution. In the more likely case of strongly anisotropic turbulence (because of strong convection) we find $n \approx 2$, i.e. an iso-angular-momentum distribution ${ }^{1}$.

\section{Settling accretion regime: observations}

Equilibrium X-ray pulsars. In equilibrium XPSRs $\left\langle\dot{\omega}^{*}\right\rangle=0$ (e.g. Vela X-1 and GX 3012). In this case, measurements of spin-up/spin-down near the equilibrium pulsar period $P_{e q}^{*}$ (or frequency $\omega_{e q}^{*}$ ) provides the additional quantity $\partial \omega^{*} / \partial \dot{M}$ (or $\partial \omega^{*} / \partial y$, where $y \equiv \dot{M} \dot{M}_{e q}$ is the mass accretion rate or X-ray luminosity normalized to the equilibrium value). In this case (see [6], [7] for more details) for the convective shell $(n=2)$ we find: a) the equilibrium NS period via binary orbital period $P_{b}$, mass accretion rate $\dot{M}$ (or X-ray luminosity $L_{x}=0.1 \dot{M} c^{2}$ ), NS magnetic field $\mu_{30}$ and relative stellar wind velocity $v_{8} \equiv v /(1000 \mathrm{~km} / \mathrm{s})$

$$
P_{e q}^{*} \approx 1300[\mathrm{~s}] \mu_{30}^{12 / 11}\left(P_{b} / 10 \mathrm{~d}\right) \dot{M}_{16}^{-4 / 11} v_{8}^{4}
$$

\footnotetext{
${ }^{1}$ If there is no convection in the shell, the magnetosphere interacts with the shell in a turbulent boundary layer. In that case the spin-down torque is $\sim \mu^{2} / R_{c}^{3}\left(R_{c}=\left(G M / \omega *^{2}\right)^{1 / 3}\right.$ is the corotation radius $)$ and is independent on $\dot{M}$. This case of weak coupling can be realized for very faint slowly rotating XPSRs.
} 
b) estimate of the coupling parameters $Z_{e q}$ or $\left(K_{1} / \zeta\right)$ via $P^{*}$ and $\partial \omega^{*} / \partial y$ :

$$
Z_{e q} \approx \frac{\left.I \frac{\partial \dot{\omega}^{*}}{\partial \dot{M}}\right|_{e q}}{\frac{4}{11} \omega^{*} R_{A}^{2}} \approx 1.8\left(\frac{\partial \dot{\omega}^{*} /\left.\partial y\right|_{y=1}}{10^{-12} \mathrm{rad} / \mathrm{s}}\right)\left(P^{*} / 100 s\right) \dot{M}_{16}^{-7 / 11} \mu_{30}^{-12 / 11}
$$

c) estimate of the NS magnetic field via $P^{*}$ and $\partial \omega^{*} / \partial y$

$$
\mu_{30, e q} \approx 5\left(\frac{\partial \dot{\omega}^{*} /\left.\partial y\right|_{y=1}}{10^{-12} \mathrm{rad} / \mathrm{s}}\right)\left(P^{*} / 100 s\right)\left(\frac{K_{1}}{\zeta}\right)^{-1} \dot{M}_{16}^{-3 / 11}
$$

d) estimate of the stellar wind velocity

$$
v_{8} \approx 0.53\left(1-z / Z_{e q}\right)^{-1 / 4} \dot{M}_{16}^{1 / 11} \mu_{30, e q}^{-3 / 11}\left(\frac{P_{*} / 100 \mathrm{~s}}{P_{b} / 10 \mathrm{~d}}\right)^{1 / 4}
$$

(note here the weak dependence on $\dot{M}$ and $\mu$ ). The observed and calculated parameters of the equilibrium wind-fed pulsars Vela X-1 and GX 301-2 are listed in Table 1. Note close values of the coupling parameter $Z_{e q} \sim 3$ (or $\zeta \sim 1 / 10$ ) for both pulsars, and the independent measurement of the stellar wind velocity similar to the observed values.

Non-equilibrium X-ray pulsars. From Eq. (2.3) it can be shown that $\dot{\omega}^{*}$ as a function of $\dot{M}$ reaches a mimimum at some accretion rate $y_{c r}=\dot{M}_{c r} / \dot{M}_{e q}=(3 / 2 n+3)^{11 / 2 n}<1$. For $n=2$ we find:

$$
\dot{\omega}_{s d, \text { max }}^{*} \approx-1.13 \times 10^{-12}[\mathrm{rad} / \mathrm{s}](1-z / Z)^{7 / 4}\left(\frac{K_{1}}{\zeta}\right) \mu_{30}^{2} v_{8}^{3}\left(\frac{P^{*}}{100 \mathrm{~s}}\right)^{-7 / 4}\left(\frac{P_{b}}{10 \mathrm{~d}}\right)^{3 / 4} .
$$

At $y<y_{c r}$ the spin-down torque should anti-correlate with the the X-ray flux variations, $\partial \dot{\omega}^{*} / \partial y<$ 0 , with $\dot{\omega}_{s d} \sim-R_{A}^{-3} \sim-\dot{M}^{6 / 11}$. This is the case observed in long-term spinning-down XPSR GX 1+4 [10], [11]. From the condition $\left|\dot{\omega}_{s d}^{*}\right| \leq\left|\dot{\omega}_{s d, \max }^{*}\right|$ we obtain the lower limit of the NS magnetic field:

$$
\mu_{30}>\mu_{30, \min }^{\prime} \approx 0.94\left|\frac{\dot{\omega}_{s d}^{*}}{10^{-12} \mathrm{rad} / \mathrm{s}}\right|\left(\frac{K_{1}}{\zeta}\right)^{-1 / 2} v_{8}^{-3 / 2}\left(\frac{P^{*}}{100 \mathrm{~s}}\right)^{7 / 8}\left(\frac{P_{b}}{10 \mathrm{~d}}\right)^{-3 / 8} .
$$

If spin-up torque can be neglected, we find another estimate of the lower limit to the NS magnetic field

$$
\mu_{30}>\mu_{30, \min }^{\prime \prime} \approx 1.66\left|\frac{\dot{\omega}_{s d}^{*}}{10^{-12} \mathrm{rad} / \mathrm{s}}\right|^{11 / 13}\left(\frac{K_{1}}{\zeta}\right)^{-11 / 13} \dot{M}_{16}^{-3 / 13}\left(\frac{P^{*}}{100 \mathrm{~s}}\right)^{11 / 13} .
$$

Note that in contrast to Eq. (3.6), this estimate is independent of the poorly known stellar wind velocity $v_{8}$ and binary orbital period $P_{b}$.

With decreasing $\dot{M}$ in non-equilibrium pulsars, the ratio of the toroidal to poloidal magnetic field components increases, reaching $B_{t} \sim B_{p}$ at $\dot{M}_{16}^{*} \approx 0.27\left|\frac{\dot{\omega}_{s d}^{*}}{10^{-12} \mathrm{rad} / \mathrm{s}}\right|^{11 / 6} \mu_{30}^{-2 / 3}$. Below this luminosity acrretion becomes more non-stationary (likely the case of GX 1+4), but it is not still centrifugally prohibited. The propeller stage begins once $R_{A}>R_{c}$ at much smaller luminosities: $\dot{M}_{16}^{* *} \approx 0.008 \mu_{30}^{3}\left(P^{*} / 100 \mathrm{~s}\right)^{-11 / 3}$.

Very slowly rotating XPSRs There are known several very slowly rotating XPSRs, including some in HMXB (SXP 1062 with $P^{*}=1062 \mathrm{~s}$ [12], 4U 2206+54 with $P^{*}=5550 \mathrm{~s}$ [13]) and some in LMXB (e.g. 3A 1954+319, $P^{*}=19400 \mathrm{~s}$ [14]). Assuming disk accretion in such pulsars would 
Table 1: Parameters of pulsars discussed

\begin{tabular}{|c|c|c|c|c|c|}
\hline Pulsars & Equili & brium & & Jon-equilibriun & \\
\hline & GX301-2 & VelaX -1 & $\mathrm{GX} 1+4$ & SXP1062 & $4 \mathrm{U} 2206+54$ \\
\hline Ref. & {$[8]$} & [9] & [11] & [12] & [13] \\
\hline & $\mathrm{Me}$ & asured paral & meters & & \\
\hline$P^{*}(\mathrm{~s})$ & 680 & 283 & 140 & 1062 & 5560 \\
\hline$P_{B}(\mathrm{~d})$ & 41.5 & 8.96 & 1161 & $\sim 300^{\dagger}$ & 19 \\
\hline$v_{w}(\mathrm{~km} / \mathrm{s})$ & 300 & 700 & 200 & $\sim 300^{\ddagger}$ & 350 \\
\hline$\mu_{30}$ & 2.7 & 1.2 & $?$ & $?$ & 1.7 \\
\hline$\dot{M}_{16}$ & 3 & 3 & 1 & 0.6 & 0.2 \\
\hline$\left.\frac{\partial \dot{\omega}}{\partial y}\right|_{y=1}(\mathrm{rad} / \mathrm{s})$ & $1.5 \cdot 10^{-12}$ & $1.2 \cdot 10^{-12}$ & $n / a$ & $n / a$ & $n / a$ \\
\hline$\dot{\omega}_{s d}^{*}$ & 0 & 0 & $-2.34 \cdot 10^{-11}$ & $-1.63 \cdot 10^{-11}$ & $-9.4 \cdot 10^{-14}$ \\
\hline & & tained paran & neters & & \\
\hline$f(u)$ & 0.53 & 0.57 & & & \\
\hline$\frac{K_{1}}{\zeta}$ & 14 & 10 & & & $\gtrsim 8$ \\
\hline Z & 3.7 & 2.6 & & & \\
\hline$B_{t} / B_{p}$ & 0.17 & 0.22 & & & \\
\hline$R_{A}(\mathrm{~cm})$ & $2 \cdot 10^{9}$ & $1.4 \cdot 10^{9}$ & & & \\
\hline$\omega^{*} / \omega_{K}\left(R_{A}\right)$ & 0.07 & 0.08 & & & \\
\hline$v_{w, \min }(\mathrm{km} / \mathrm{s})$ & 500 & 740 & & & \\
\hline$\mu_{30, \min }$ & & & $\mu_{\min }^{\prime} \approx 4$ & $\mu_{\min }^{\prime \prime} \approx 20$ & $\mu_{\min }^{\prime} \approx 3.6$ \\
\hline
\end{tabular}

$\dagger$ Estimated from the Corbet diagram

$¥$ Typical velocity assumed in Be X-ray binaries

require incredibly high NS magnetic fields (see e.g. discussion in [15]). However, application of our model to these and other non-equilibrium XPSRs (see Table 1) gives the low limits of the NS surface magnetic field in the usual range $10^{12}-10^{13} \mathrm{G}$, and it is too preliminary to classify these objects as accreting magnetars. Note also that if at small X-ray luminosities convection is not developed in the shell, a quasi-Keplerian rotation law with $n=3 / 2$ can be established. The equilibrium NS spin period in this case is $P_{e q}^{(n=3 / 2)}=P_{e q}^{(n=2)} \sqrt{R_{B} / R_{A}} \sim 10 P_{e q}^{(n=2)}$. That means that NS periods in such XPSRs can easily reach a few $10000 \mathrm{~s}$ for the standard NS magnetic field.

Other applications A possible implication of the settling accretion theory can be for nonstationary phenomena in XPSRs. The theory explains the observed temporary 'off'-states in Vela $\mathrm{X}-1$, GX 301-2, 4U 1907+09, when the plasma cooling near the magnetospheric equator occurs due to radiative processes [16]. The Compton cooling turns out to be ineffective due to X-ray pattern changing from fan-beam to the pencil-beam (as suggested by the observed X-ray pulse shape changes during the off-state in Vela X-1 [17]).

A dynamical instability of the shell on the time scale of the order of the free-fall time from the magnetosphere can appear due to increased Compton cooling and hence increased mass accretion 
rate in the shell, leading to an X-ray outburst with duration lasting about the free-fall time scale of the entire shell $(\sim 1000 \mathrm{~s})$. Such a transient behaviour is observed in supergiant fast X-ray transients (SFXTs) (see e.g. [18]), in which slow X-ray pulsations are found (e.g. IGRJ16418-4532, $P^{*} \approx 1212 \mathrm{~s}$ [19]). The observed flaring behavior can be the manifestation of a Rayleigh-Taylor instability from the magnetospheric radius occurring on the dynamical time scale $\sim R_{A}^{3 / 2} / \sqrt{G M}$.

\section{Conclusion}

At X-ray luminosities $<4 \times 10^{36} \mathrm{erg} / \mathrm{s}$ wind-fed X-ray pulsars can be at the stage of subsonic settling accretion. In this regime, the accretion rate onto NS is determined by the ability of plasma to enter magnetosphere via Rayleigh-Taylor instability. The angular momentum can be transferred through the quasi-static shell via large-scale convective motions initiating turbulence cascade. The theory explains the long-term spin-down in wind- fed accreting pulsars and properties of shortterm torque-luminosity correlations. Long-period low-luminosity X-ray pulsars are most likely experiencing settling accretion too. Spectral and timing measurements of slowly rotating X-ray pulsars can be used to further test this accretion regime.

\section{References}

[1] E. Bozzo, M. Falanga and L. Stella, ApJ 6831031 (2008).

[2] J. Arons and S. Lea, ApJ 207914 (1976).

[3] D.J. Burnard, J. Arons and S.M. Lea, ApJ 266175 (1983).

[4] R. F. Elsner and F. K. Lamb, ApJ 215897 (1977).

[5] M.E. Davies and J.E. Pringle, MNRAS 196209 (1981).

[6] N.I. Shakura, K.A. Postnov, A.Yu. Kochetkova and L. Hjalmarsdotter, MNRAS 420216 (2012).

[7] N.I. Shakura, K.A. Postnov, A.Yu. Kochetkova and L. Hjalmarsdotter, Physics-Uspekhi, in press (2013); arXiv:1302.0500.

[8] V. Doroshenko et al., $A A 515$ A10 (2010).

[9] V. Doroshenko, PhD Thesis, University of Tübingen (2011).

[10] D. Chakrabarty, L. Bildsten, M.H. Finger, et al., ApJ 481 L101 (1997).

[11] A. González-Galán, E. Kuulkers, P. Kretschmar, et al., AA 537 A66 (2012).

[12] F. Haberl et al $A A \mathbf{5 3 7}$ L1 (2012).

[13] P. Reig, J.M. Torrejón, P. Blay, MNRAS 425529 (2012).

[14] D.M. Marcu, F. Fürst, K. Pottschmidt, et al., ApJ 742 L11 (2011).

[15] W. Wang, arXiv:1211.5214 (2012)

[16] N.I. Shakura, K.A. Postnov, and L. Hjalmarsdotter, MNRAS 428670 (2013).

[17] V. Doroshenko, A. Santangelo, V. Suleimanov, AA 529 A52 (2011).

[18] L. Ducci, L. Sidoli and A. Paizis, MNRAS 4081540 (2010).

[19] L. Sidoli, S. Mereghetti, V. Sguera and F. Pizzolato, MNRAS 420554 (2012). 